\title{
Do Chamomile effect on duration, amount of bleeding, and interval of menstrual cycles?
}

\author{
Elham Najafi Mollabashi' ${ }^{1}$ Tahereh Ziaie², Reza Bekhradi ${ }^{3}$, Zahra Bostani Khalesi²* \\ ${ }^{1}$ Student Research Committee, Guilan University of Medical Sciences, Rasht, Iran \\ ${ }^{2}$ Social Determinants of Health Research Center, Guilan University of Medical Sciences, Rasht, Iran \\ ${ }^{3}$ Department of the Development and Coordination of Scientific Texts, Barij Herbal Plant Research Center, Kashan, Iran
}

\section{Key Words}

Menstruation, bleeding, Chamomile, Herbal Plants

\begin{abstract}
Objectives: Heavy menstrual bleeding has a significant effect on women's daily life and health consequences for theirs. Attention to women's and girls' menstrual health is critical for their health. This study presents the results of a novel research on the effect of chamomile capsule on the amount of bleeding, and the duration and interval of menstrual cycles.
\end{abstract}

Methods: The current study was a randomized, double-blind, clinical trial, which was carried out on 118 female students living in dorms of Guilan University of Medical Sciences. The participants were divided into two groups of 59, each receiving either chamomile capsule $250 \mathrm{mg}$ or placebo three times a day, for an interval beginning from 7 days before the starting menstruation till the next onset. Higham chart is the pictorial blood loss assessment to determine the amount and duration of menstrual bleeding. Data were analyzed using SPSS version 20. Alpha was set at 0.05 for all analyses

Results: The average amount of bleeding in the chamomile group decreased after taking the capsule $(\mathrm{p}=$

Received: May 27, 2019 Reviewed: Dec 23, 2019 Accepted: Feb 17, 2020

(0) This is an Open-Access article distributed under the terms of the Creative Common Attribution Non-Commercial License (http://creativecommons.org/licenses/by-nc/4.0/) which permits unrestricted noncommercial use, distribution, and reproduction in an medium, provided the original work is properly cited.
0.001). However, statistical tests did not show any significant difference in terms of duration and interval between two bleeding in both the experimental and control groups $(\mathrm{p}>0.05)$.

Conclusion: The present study showed that the chamomile capsule decreases the amount of menstrual bleeding and can be used as a therapeutic method.

Conclusion: The present study showed that the chamomile capsule decreases the amount of menstrual bleeding and can be used as a therapeutic method.

\section{Introduction}

Menstruation is a physiological process that occurs in most women of reproductive age. This is the regular discharge of blood and mucosal tissue from the inner lining of the uterus through the vagina [1], that occurs due to the declining levels of progesterone and estrogen which stimulates the release of prostaglandins that cause the uterine spiral arterioles to constrict [2]. As a result, the cells they supply become oxygen-deprived and start to die. The entire stratum functional is sloughed off [3]. The menstrual flow passes from the uterine cavity through the cervix and vagina to the exterior [4]. Menstruation occurs according to a specific pattern and order, the menstrual pattern includes the amount of bleeding and duration and interval of menstrual cycles [3]. The duration of the menstrual cycle typically ranges from 24 to 35 days, with an average of 28 days. The menstrual phase, which is generally called menstruation or menses, lasts for about the first 5 days of the cycle [5], and the average bleeding in the 
normal menstrual cycle is $30-40 \mathrm{ml}$ [6]. It seems that the use of some herbs can affect menstruation. Today, the use of herbal medicine has become more important. In Iran, more attention has been paid to traditional medicine [7]. One of the most well-known and most used herbal plants is Matricaria Chamomilla (Chamomile) [8]. It has been stated in traditional books that chamomile is a medicinal plant that can affect menstruation [9]. Chamomile is a plant of the Asteraceae Family, Matricaria Genus, and Chamomilla Species [10]. Chamomile extract consists of 120 types of chemical composition including Chamazulene which has anti-inflammatory and antioxidant effects, Apigenin with anti-inflammatory, sedative and anti-neoplastic effects, Flavonoid with anti-inflammatory and anti-anxiety effects, and Alpha-Bisabol with anti-inflammatory and digestive effects, and the most important active ingredients in it are: $\alpha$ - Hetroside, B- Hetroside, Salicylic acid, and Metoxycoumarin, Chamomile has a strong anti-spasmodic effect due to the presence of a spirometer [11]. Chamomile antispasmodic properties justify the ancient reputation of this plant in relieving painful menstruation [12]. The Glycine chemical found in chamomile relieves muscle spasms and can act as a relaxant, higher levels of Glycine may relax the uterus [13]. In a study by Sharifi et al in 2014, excessive menstrual bleeding was reported in the chamomile extract receiving group [12]. The results of Ehsani et al. research in 2014 showed that a decoction of herbal plants (Anthemis, Salvia, and Zataria) could decrease the intensity of menstrual bleeding [14]. Although, the United States Food and Drug Administration has classified chamomile essential oil and oil as safe items [8]. But, Chamomile is of the Compositae family; thus, patients hypersensitive to this family may be experience allergy symptoms. Also, possible side effects of chamomile include severe allergic reactions (chest tightness, wheezing, hives, rash, itching), contact dermatitis/skin reactions, eye irritation (when applied near the eyes), hypersensitivity reaction and vomiting [15]. In the present study, we have been using chamomile capsules, which is a new form of this plant and made from dried flowers of chamomile and containing all the active ingredients of chamomile. Considering the willingness of people to receive herbal medicines, it is imperative that physicians and midwives have full information about the effect of herbal medicines on the menstrual pattern. The literature review indicates that Chamomile's effect on menstrual bleeding was different. Therefore, in order to consolidate the previous results or find new results, this study aimed to investigate the effect of chamomile capsule on the amount of bleeding and the duration and interval of menstrual cycles.

\section{Materials and methods}

This randomized, double-blinded and placebo-controlled trial was performed following the approval by the ethics committee of Guilan University of Medical Sciences, Guilan, Iran, under registration (IR.GUMS.REC.1395.396) and recorded by the International Center for Registration of Clinical Trials in Iran (code IRCT201705214295N3). The study was conducted on 118 students residing in the Kows- ar dormitory of Guilan University of Medical Sciences. The inclusion criteria were as follows: being single, regular menstrual cycles of 21 - 35 days, no physical or psychological ill conditions, not on medication (hormones, vitamins, herbal, anti-depressant, aspirin, or Warfarin), no history of allergy to herbal drugs, no surgical operation during the last six months, not being a professional athlete. Those with the above-mentioned criteria were entered into the study. Informed written consent was also given by each participant. Exclusion Criteria: The exclusion criteria included: improper use of capsules, allergy to drugs, unwillingness to continue participation in the study and receiving and taking medications during the study. 118 students entered into the study, then, participants were randomly divided into 2 equal groups of intervention (N: 59) and control (N: 59).

Tools for data collection were: Pictorial blood loss assessment charts. The women were asked to record the number of pads and tampons used and the degree to which individual items were soiled with blood. The score calculated by multiplying the number of slightly, moderately, and heavily soiled pads and tampons by different fixed factors (respectively, one, five, and 20 for pads and one, five, and 10 for tampons). Small clots score one point, and large clots, five points. In doing so, Total scores were calculated, and based on the amount of the overall score of menstrual flow were evaluated. The women also were asked to indicate the duration of flows and cycles. The demographic questionnaire: It consisted of 3 demographic items about their age, weight, height and 3 questions related to menarche, duration of menses, and menstrual interval. Content validity was used to assess the validity of the tools. In doing so, the questionnaires were prepared based on the research goal and relevant scientific sources and consultation with ten experts. The required revisions and changes were made to the questionnaire, as suggested by the expert reviewers.

Once the definitive diagnosis of the amount and duration of menstrual flow was made, the participants were randomly divided into two groups. Chamomile group: The participants received Chamomile capsule received 250 $\mathrm{mg}$, and the placebo group received a placebo capsule, all participants received capsules from 7 days prior to menstrual bleeding till the next onset of menstruation period, three times daily for one cycle.

The Chamomile and placebo capsules were prepared with an identical appearance by the Barij Essence pharmaceutical company (Kashan, Iran. Chamomile powder as the drug was prepared through drying the aerial organ of the plant, and capsules containing $250 \mathrm{mg}$ of the obtained powder were prepared. The starch powder as a placebo was filled in oral capsules. The two types of capsules were put in separate coded packs by the pharmacy and participants were blinded to the type of the ingredients.

Once the questionnaires were collected following each period, the respective data were obtained and analyzed by statistical tests. SPSS version 23 was used along with descriptive statistical techniques such as frequency table, means values, the standard deviation to evaluate the efficacy of the treatments and associated side effects on the recipients. Inferential statistical tests such as independent t, paired sample t-test, Wilcoxon signed-rank test, Chi- 
square, Mann Whitney test was used to make intergroup and intragroup comparisons in terms of intensity of the amount and duration of menstrual bleeding. A P-value of less than 0.05 was considered significant.

\section{Results}

Baseline characteristics of cases and controls are presented in Table 1, the results showed that $73 \%$ of the first group and $68 \%$ of the second group ranged between 20 - 25 years, the age at menarche in $84 \%$ of the first group and $87 \%$ of the second group was 11 - 13 years old, $63 \%$ of the first group and $56 \%$ of the second group had normal body mass index, the duration of bleeding in $48 \%$ of the first group and $56 \%$ of the second group was 5 - 6 days, and the interval between the periods was $52 \%$ the first group and $56 \%$ in the second group was 24 - 27 days. The rate of bleeding was estimated at more than $60 \mathrm{cc}$ in $43 \%$ of the subjects in the first group and $39 \%$ of the second group. The two groups were matched together and there was not significantly differ between the Chamomile and control groups ( $p>0.05)$. As shown in Table 2, in the Chamomile group amount of bleeding after intervention reduced significantly than before intervention from $56.9 \pm 13.4$ to $52.5 \pm 12.8(\mathrm{p}<0$. 001 ), whereas this score after intervention in the placebo group had no significant difference with before intervention $(55.7 \pm 12 / 2$ to $54.4 \pm 12 / 8, p=0.36)$. However, duration and interval of menstrual cycles after intervention in Chamomile and the placebo group had no significant difference with before intervention ( $p>0.05)$, also, there was no significant difference between the d duration and interval of menstrual cycles after treatment between the two groups $(p>0.05)$.

\section{Discussion}

Based on our results, after having one menstrual cycle intervention, participants in the Chamomile group showed significant reductions in the amount of bleeding than before the intervention.

One of the most likely factors causing severe menstrual bleeding has been reported to be an increase in the production of uterine prostaglandins $[6,16]$. Regarding the fact that E2 and F2a prostaglandins increase blood flow to the uterus and E2 prostaglandins are Vasodilators, the probability of an increase in menstrual bleeding increases [17]. Several research has shown that the inhibition of prostaglandin synthesis can ameliorate the amount of menstrual bleeding in women $[18,19]$. In the present study, the amount of menstrual bleeding in the pre-intervention stage was $56 \mathrm{mg}$ on average, which was more than the mean of bleeding in the normal menstrual cycle (40-30 $\mathrm{ml}$ ) per cycle [2]. Since chamomile has anti-prostaglandin property, this plant interrupts Cyclooxygenase resulting in the production of prostaglandins and leukotrienes [11]. Considering the difference between the results

Table 1 Characteristics of the participants

\begin{tabular}{lccc}
\hline Variables & $\begin{array}{c}\text { Chamomile } \\
(\mathbf{n}=\mathbf{5 4})\end{array}$ & $\begin{array}{c}\text { Placebo } \\
(\mathbf{n}=\mathbf{5 4})\end{array}$ & P-value \\
\hline Age (years) & $22.8 \pm 2.1$ & $23.5 \pm 2.3$ & $0.09^{*}$ \\
Body mass index (kg/m2) & $21.7 \pm 1.9$ & $22.2 \pm 2.0$ & $0.25^{*}$ \\
Menarche age (years) & $12.1 \pm 0.9$ & $11.8 \pm 0.9$ & $0.07^{*}$ \\
Duration of menses (days) & $6.5 \pm 0.6$ & $6.3 \pm 0.7$ & $0.12^{*}$ \\
Menstrual interval (days) & $27.2 \pm 1.5$ & $26.6 \pm 1.2$ & $0.05^{*}$ \\
\hline * In depended t- test & & &
\end{tabular}

* In depended t- test

Table 2 Amount and duration of menstrual bleeding before and after treatment

\begin{tabular}{lcccc}
\hline \multirow{2}{*}{ Variables } & Group & $\begin{array}{c}\text { Before } \\
\text { treatment } \\
\text { (Mean } \pm \mathrm{SD})\end{array}$ & $\begin{array}{c}\text { After treatment } \\
(\text { Mean } \pm \mathrm{SD})\end{array}$ & P-value* \\
& Chamomile & $56.9 \pm 13.4$ & $52.5 \pm 12.8$ & $\mathrm{P}<0.001$ \\
\hline $\begin{array}{l}\text { Amount of menstrual } \\
\text { bleeding(cc) }\end{array}$ & Placebo & $55.7 \pm 12.2$ & $54.4 \pm 12.8$ & $\mathrm{P}=0.36$ \\
\hline $\begin{array}{l}\text { Duration of menstrual } \\
\text { bleeding(day) }\end{array}$ & Chamomile & $6.3 \pm 0.7$ & $6.5 \pm 0.6$ & $\mathrm{P}=0.08$ \\
\hline $\begin{array}{l}\text { Interval of menstrual } \\
\text { cycles(day) }\end{array}$ & Placebo & $6.3 \pm 0.5$ & $6.3 \pm 0.6$ & $\mathrm{P}=0.48$ \\
\hline
\end{tabular}

* paired sample t-test; ** In depended t- test 
of this study with Sharifi's research, which showed that Chamomile extract increases menstrual bleeding [12], it seems that the type and concentration of chemical compounds present in dry chamomile and chamomile extract have different effects on menstruation. Few studies have been performed on the dose and duration of chamomile for the treatment of the amount of bleeding, and duration and interval of menstrual cycles. But, the evidence available indicates the extract of chamomile at the doses of $25 \mathrm{ml}$ decreased the amount of menstrual bleeding [14]. Also, a typical protocol for decrease the severity of dysmenorrhea taking Chamomile at the doses of $15 \mathrm{ml}$ [20]. The results of the study by Karimian et al. revealed the chamomile capsule effect on the decrease amount of menstrual bleeding. In the study, participants consumed $250 \mathrm{mg}$ chamomile capsule every 8 hours in the first 3 days of menstruation [21]. As well as, in contrast to the research conducted by Ehsani et al. With the aim of investigating the efficiency of herbal medicine (Anthemis, Salvia, and Zataria) on menstrual cycle, blood discharging [14], it seems that the consumption of dried chamomile capsules, such as drinking mixture decoction of the herbal composition, reduces the amount of menstrual bleeding. Similarly, in a study by Mirbagheri et al. (2012), results similar to those of the present study were obtained, based on the results of this study pure honey consumption has reduced the amount of menstrual bleeding in comparison with before the intervention, but it did not affect the duration of bleeding and the interval between two bleeding [22]. Also, in a study by Mohammad Alizadeh et al. The positive effect of calcium and magnesium composition for reducing the amount and duration of menstrual bleeding has been shown [23]. In the above studies, chamomile extract and tea or chemical compounds were used to reduce bleeding, but in the present study, chamomile capsules were used to reduce bleeding.

\section{Conclusions}

Because herbal medicines are generally considered safe and proved to be effective against various human ailments, have received attention in different fields of medical science in the past decade, and due to the increasingly popular tendency to use herbal medicines, it is imperative that physicians and midwives be aware of the effect of these herbs on menstrual patterns. The results of our study demonstrated the usefulness and benefits of chamomile in reducing the amount of menstrual bleeding, while no specific side effects have been seen.

Several limitations exist in the current study beginning with the sample being drawn from a population of students at a single dormitory. Thus, the results may not be generalizable to students at other demographics. Secondary, the bleeding patterns were self-reported by the participants, they may not be able to assess the amount of menstrual bleeding accurately. Accordingly, the major implication of the present research is that it provides useful information for physicians. They can prescribe Chamomile capsules as a traditional medicine with beneficial effects in reducing menstrual bleeding. Chamomile is less toxic or having fewer side effects in contrast to synthetic drugs.

\section{Acknowledgments}

This paper is a part of the midwifery master's degree thesis. The authors thank the social determinants of the health research center of Guilan University of medical sciences, and Kowsar dormitory administrators. We also thank the Barij Essen Kashan, pharmaceutical company for their contributions in study drug preparation and dispensing. Sincere thanks go to all the students who participated in this study.

\section{References}

1. Kocaoz S, Cirpan R, Degirmencioglu AZ. The prevalence and impacts heavy menstrual bleeding on anemia, fatigue and quality of life in women of reproductive age. Pak J Med Sci. 2019;35(2):365-70.

2. Reed BG, Carr BR. The Normal Menstrual Cycle and the Control of Ovulation. Endotext [Internet]. MDText. com, Inc.; South Dartmouth (MA): Aug 5, 2018.

3. Munro MG, Critchley HOD, Fraser IS., FIGO Menstrual Disorders Committee. The two FIGO systems for normal and abnormal uterine bleeding symptoms and classification of causes of abnormal uterine bleeding in the reproductive years: 2018 revisions. Int J Gynaecol Obstet. 2018;143(3):393-408.

4. Monis CN, Tetrokalashvili M. Menstrual Cycle Proliferative and Follicular Phase. [Updated 2019 May 18]. In: StatPearls [Internet]. Treasure Island (FL): StatPearls Publishing; 2019 Jan-. Available from: https://www. ncbi.nlm.nih.gov/books/NBK542229/

5. Wilbur J, Torondel B, Hameed S, Mahon T, Kuper H. Systematic review of menstrual hygiene management requirements, its barriers and strategies for disabled people. PLoS One. 2019;14(2):e0210974.

6. Sriprasert I, Pakrashi T, Kimble T, Archer DF. Heavy menstrual bleeding diagnosis and medical management. Contracept Reprod Med. 2017;2:20.

7. Zargari A. Herbal Plants. 5th ed. Tehran University Press. Tehran, 1991: 553-62.

8. Shang NC. The efficacy of a physiotherapeutic complex (Angelica Sinensis, Dioscorea Villosa, Matricaria Chamomilla, Viburnum opulus and Zingiber Officinalis) compared with homeopathic Similimum in the treatment of primary dysmenorrheal [technology, homeopathy MA Thesis]. Durban: Faculty of Health Sciences at the Durban University of Technology; 2015.

9. Saghafi N, Rhkhshandeh H, Pourmoghadam N, Pourali L, Ghazanfarpour M, Behrooznia A, et al. Effectiveness of Matricaria chamomilla (chamomile) extract on pain control of cyclic mastalgia: a double-blind randomized controlled trial. J Obstet Gynaecol. 2018;38(1):81-4.

10. Al-Dabbagh B, Elhaty IA, Elhaw M. Antioxidant and anticancer activities of chamomile (Matricaria recutita L.). BMC Res Notes. 2019;12(1):3.

11. Singh O, Khanam Z, Misra N, Srivastava MK. Chamomile (Matricaria chamomilla L.): An overview. Pharmacogn Rev. 2011;5(9):82-95.

12. Sharifi F, Mojab F, Simbar A. comparative study of the effects of Matricaria Chamomile extract and mefenamic acid on the severity of premenstrual syndrome 
symptoms. Arak Medical University Journal (AMU). 2013;16(70): 71-8.

13. McKay DL and Blumberg JB. A review of the bioactivity and potential health benefits of chamomile tea. Phytotherapy Research. 2006;20 (7): 519-30.

14. Ehsani P, Nazayer H, Memari A. The efficiency of herbal medicine (Anthemis, Salvia, and Zataria) on Menstrual Cycle Blood Discharging. Journal of Women and Culture. .2014;4(18):127-40.

15. Wu Y-n, Xu Y and Yao L. Anti-inflammatory and Anti-Allergic Effects of German Chamomile. Journal of Essential Oil Bearing Plants. 2011;14 (5): 549-58.

16. Bofill Rodriguez M, Lethaby A, Low C, Cameron IT. Cyclical progestogens for heavy menstrual bleeding. Cochrane Database Syst Rev. 2019 Aug 14;8:CD001016.

17. Jamieson MA. Disorders of menstruation in adolescent girls. Pediatr Clin North Am. 2015;62(4):943-61.

18. Bofill Rodriguez M, Lethaby A, Farquhar C. Non-steroidal anti-inflammatory drugs for heavy menstrual bleeding. Cochrane Database Syst Rev. 2019;9:CD000400.

19. Rod J. Flower. The development of COX2 inhibitors. Nature Reviews Drug Discovery 2003; 2:179-91.

20. Sharghi M, Mansurkhani SM, Larky DA, et al. An update and systematic review on the treatment of primary dysmenorrhea. JBRA Assist Reprod. 2019;23(1):51-7.

21. Karimian Z, Sadat Z, Bahrami N, Kafaie M. Comparison of chamomile and mefenamic acid capsules in hemorrhage of menstruation. Iranian Journal of Obstetrics, Gynecology and Infertility. 2015;18(157):11-7.

22. Mirbagher AN, Hafezi M, Salehi S, Zahtabchi S. Comparing the effect of pure and impure honey on severity of pain, amount of bleeding, and duration and interval of menstrual cycles in female students with primary dysmenorrhea. Evidence-Based Eye Care. 2012;2(1):23-33

23. Mohammad Alizadeh S, Mirghafourvand M, Javadzadeh Y, Nezamivand S. Effect of Calcium with and without Magnesium on amount and duration of menstrual bleeding in students with primary dysmenorrhea. IJOGI. 2014; 16(83):1-8. 